\title{
Grupos Focais Online e Pesquisa em Psicologia: Revisão de Estudos Empíricos entre 2001 e $2011^{1}$
}

\author{
Gabriela Sagebin Bordini * \& Tania Mara Sperb \\ Universidade Federal do Rio Grande do Sul, Porto Alegre, Brasil
}

\begin{abstract}
RESUMO
O grupo focal é uma técnica de coleta de dados muito utilizada em pesquisa, predominantemente, no seu formato presencial. Nas últimas décadas, alguns grupos focais presenciais vêm migrando para o ambiente virtual. No entanto, a realização dos grupos focais por meio do uso de computadores e da internet gera uma série de especificidades. O objetivo deste trabalho é investigar e discutir como os pesquisadores do campo da psicologia têm utilizado os grupos focais online em seus estudos empíricos. A partir de uma revisão de artigos publicados entre 2001 e 2011, apresentam-se recomendações para o emprego dessa técnica, baseadas nas diversas particularidades relatadas pelos pesquisadores.
\end{abstract}

Palavras-chave: grupos focais online; grupos focais virtuais; internet; métodos de pesquisa; psicologia.

\section{ABSTRACT \\ Online Focus Groups and Psychological Research: A Review of Empirical Studies Between 2001 and 2011}

Focus group is a technique of data collection often used in research, usually in the face-to-face format. In recent decades, a part of such face-to-face focus groups has migrated to the virtual environment. However, conducting focus groups using computers and internet generates a number of specificities. This paper aims to investigate and discuss the use of online focus groups in psychological empirical research. Through a review of articles published between 2001 and 2011, we present recommendations for using this technique, drawn upon several peculiarities reported by the researches.

Keywords: online focus groups; virtual focus groups; internet; research methods; psychology.

Este é um estudo de revisão, uma pesquisa em que se explora a produção científica sobre certo tema, em um dado período. O objetivo deste trabalho é investigar e discutir como os grupos focais online vêm sendo utilizados em pesquisas empíricas recentes no campo da psicologia e relatar suas especificidades. Com isso, busca-se apresentar algumas diretrizes para o uso dessa nova técnica, contribuindo para a sua divulgação nesse campo da ciência.

O grupo focal é uma técnica de coleta de dados consagrada. Trata-se, basicamente, de uma discussão direcionada, em grupo de seis a doze componentes, acerca de um tópico específico, com a duração de pelo menos 1 hora e não mais de 2 horas (Sirihal Duarte,
2007). Para facilitar o debate e manter o foco dos participantes no tópico há um moderador, geralmente o próprio pesquisador, que se responsabiliza também por elaborar um guia de entrevista, relatar e analisar seus resultados (Owen, 2001; Sirihal Duarte, 2007). O moderador preocupa-se com as relações entre os componentes e com o processo de discussão (Abreu, Baldanza, \& Gondim, 2009), que podem levar à reelaboração dos pontos de vista dos participantes (Stjernsward \& Ostman, 2011; Tates et al., 2009). Por conta disso, os grupos focais são utilizados para provocar discussões em profundidade (Chase \& Alvarez, 2000) e acessar dados que seriam mais difíceis de encontrar sem a interação grupal (Morgan, 1997).

\footnotetext{
Endereço para correspondência: Gabriela Sagebin Bordini - charlestonbordini@yahoo.com.br
} 
Os grupos focais têm sido bastante utilizados na investigação de características de uma determinada população e dos pontos de vista de um pequeno número de participantes, sobretudo em pesquisas qualitativas (Schneider, Kerwin, Frechtling, \& Vivari, 2002) e que ocorrem presencialmente. Hoje em dia, contudo, com o advento do contexto virtual de interação, os grupos focais no formato online vêm sendo cada vez mais citados na literatura científica como técnica de coleta de dados (Fox, Morris, \& Rumsey, 2007a).

Os grupos focais online - também chamados de grupos focais virtuais, mediados por computador ou eletrônicos - são grupos focais que têm lugar na internet, nos quais os participantes e o moderador se comunicam pelo computador. Assim como nos grupos presenciais, no modelo online o moderador segue um guia de entrevista elaborado anteriormente e estimula a interação entre os participantes, para que discutam o tópico proposto. Alguns programas de computador possibilitam a comunicação através de imagem e som, com o uso de câmeras e microfones. No entanto, é mais comum que a discussão se dê por escrito, com os membros do grupo escrevendo suas mensagens em uma caixa de texto localizada, geralmente, na parte de baixo da tela. As mensagens e a identificação do participante que as enviou aparecem na tela de cada membro, conforme vão sendo postadas. É possível ler as mensagens enviadas anteriormente clicando em um comando especifico para isso (Bordini \& Sperb, 2011; Chase \& Alvarez, 2000; Schneider et al., 2002).

Os grupos focais online em que a comunicação ocorre por escrito dividem-se em síncronos e assíncronos. Nos grupos focais online síncronos, a comunicação entre os participantes é simultânea e a troca de mensagens entre eles acontece em salas de bate-papo virtuais (chat) ou por meio de programas de computador que permitem a realização de conferências virtuais, como o MSN (Sirihal Duarte, 2007). Os grupos focais assíncronos, por outro lado, são grupos em que a discussão não se dá em tempo real; os membros podem opinar e ler os comentários dos outros em qualquer momento (Sirihal Duarte, 2007). Neste tipo de grupo focal online, a comunicação entre os participantes ocorre pela simples troca de $e$-mails, ou por meio de fóruns de discussão ou listas de discussão virtuais. Os fóruns são acessados pelos participantes mediante um endereço fornecido pelo pesquisador, já as listas de discussão consistem em um elenco de endereços de $e$ mails englobados em apenas um, para o qual se envia uma mensagem que será recebida por todos os membros da lista.
Em sua origem, os grupos focais online eram usados pela área de marketing para coletar ideias (Chase \& Alvarez, 2000). Na medicina e na enfermagem, são bastante utilizados para a realização de grupos de acompanhamento, de apoio e de troca de sentimentos acerca de diversas condições patológicas. Já como técnica de coleta de dados, os grupos focais online estão se sobressaindo na área da enfermagem, das ciências sociais e do marketing. Também na psicologia, referências ao seu uso vêm se tornando mais frequentes na literatura científica (Fox et al., 2007a), ainda que essa informação refira-se apenas às publicações em língua inglesa. No Brasil, embora a comunicação com outras pessoas seja o principal motivo de acesso à internet (IBGE, 2008), até o ano de 2009, os estudos que utilizavam grupos focais online "praticamente inexistiam na pesquisa qualitativa em psicologia" (Bordini \& Sperb, 2011, p. 437). Ou seja, essa é uma técnica relativamente nova e pouco difundida no campo psicológico, no Brasil.

Estudos que compararam o modelo online com o presencial encontraram semelhanças, mas também diferenças. Sirihal Duarte (2007) observou similaridades quanto ao conteúdo das opiniões obtidas e aos procedimentos de análise dos dados coletados. Já Chase e Alvarez (2000), que compararam o processo envolvido nos dois modelos, notaram que havia semelhança na forma dos grupos focais presenciais e online. Essas pesquisas, no entanto, ressaltaram as particularidades quanto ao processo de comunicação nestas duas técnicas de grupo focal. Sirihal Duarte, por exemplo, mencionou a discrepância na linguagem empregada pelos participantes nos dois modelos. Como concluíram Kralik, Price, Warren e Koch (2006), o acesso aos dados através da comunicação mediada por computador em pesquisa qualitativa tem características e desafios diferentes daqueles presentes nos modelos mais tradicionais de coleta de dados.

Isso mostra que os grupos focais online apresentam uma série de particularidades, não consistindo em uma simples transposição dos grupos presenciais para o contexto virtual (Abreu et al., 2009; Bordini \& Sperb, 2011; Fox et al., 2007a). Assim, é importante investigar que uso a pesquisa em psicologia tem feito dos grupos focais online e quais as especificidades relatadas pelos investigadores que têm se utilizado dessa técnica.

\section{MÉTODO}

Realizou-se uma busca em bases de dados vinculadas à psicologia e a áreas afins: (a) PsycArticles, (b) 
PsycINFO, (c) Scientific Electronic Library Online, e (d) Periódicos Eletrônicos em Psicologia. Para complementar, utilizou-se o portal da Biblioteca Virtual de Saúde Brasil, que procura em diversas bases de dados. O descritor grupo focal deveria estar presente no título, no resumo ou nas palavras-chave dos artigos, acompanhado de qualquer um dos seguintes descritores: (a) online, (b) virtual, (c) eletrônico, (d) web, (e) computador, e (f) internet. As buscas foram executadas em português e em inglês e restringiram-se ao período entre 2001 e 2011, pois a técnica dos grupos focais é relativamente recente.

Do total de trabalhos reunidos, selecionaram-se apenas aqueles classificados como artigos, publicados em periódicos revisados por pares. Além disso, foram considerados somente os trabalhos empíricos que descreviam o uso de algum grupo focal online, conduzido por escrito, utilizado para coletar dados. Excluíram-se os artigos que não estivessem disponíveis online, na íntegra.

Os artigos foram, então, agrupados conforme o tipo de grupo focal online relatado - síncrono ou assíncrono - por se considerar que são técnicas diferentes e que, portanto, seguiriam procedimentos distintos. Após essa divisão, leram-se todos os artigos na íntegra para que os grupos focais online neles descritos fossem examinados conforme os seguintes eixos de análise: (a) ferramentas - o programa que foi utilizado para a realização do grupo focal e os dispositivos de segurança que oferecia; (b) participantes - meios pelos quais foram recrutados e pelos quais devolveram o termo de consentimento livre e esclarecido (TCLE), possibilidade de se reunirem se o grupo focal não fosse online, e tamanho da amostra; (c) moderação do grupo - número de moderadores e sua relação com a pesquisa; (d) natureza dos tópicos discutidos; (e) equilíbrio entre as participações dos membros do grupo; e (f) anonimato dos participantes. Esses eixos foram desenvolvidos com base, principalmente, em Abreu et al. (2009), Bordini e Sperb (2011) e Fox et al. (2007a), que citaram várias particularidades dos grupos focais online.

\section{RESULTADOS E DISCUSSÃO}

Encontraram-se 46 artigos. Quase a metade destes (22 artigos) fora publicada em revistas de ciências humanas ou da saúde não especificamente do campo da psicologia, e não tinha nenhum psicólogo como autor. No entanto, optou-se por mantê-los na amostra para aumentar o corpus analisado. Identificaram-se 30 artigos que relatavam uso de grupos assíncronos e 16 que relatavam uso de grupos síncronos. Esses dois grupos de artigos foram examinados em separado. A seguir, apresentam-se os resultados da análise dos artigos.

\section{Grupos Focais Online Assíncronos}

A maior parte dos artigos $(65,2 \%)$ relatava grupos focais online assíncronos.

\section{Ferramentas utilizadas}

Este eixo de análise refere-se (a) aos tipos de programas de computador por meio dos quais foram realizados os grupos focais online e (b) aos seus dispositivos de segurança. O tipo de programa de computador mais utilizado para a execução dos grupos focais online assíncronos foi o fórum virtual, citado em 24 artigos. Os fóruns online são acessados por meio de um endereço virtual e permitem que a discussão seja dividida em tópicos. Além dos fóruns, três artigos referiram o uso de listas de $e$-mails. Nestas todas as mensagens vão se acumulando na caixa de entrada de cada membro da lista, uma após a outra na ordem em que foram enviadas pelos participantes. Um artigo não especificou o tipo de ferramenta usada para realizar o grupo focal. Também foi referido, em dois artigos, o uso de programas especificamente desenvolvidos para a realização de discussões virtuais.

A marca ou o fornecedor do programa escolhido para a condução dos fóruns e listas não são especialmente importantes, mas a maior parte dos pesquisadores optou por programas hospedados pelo site da sua universidade ou pelo site do seu projeto de pesquisa. O fundamental é que o programa possua um modelo de segurança que impeça o acesso de não convidados à discussão e que permita que a administração da lista ou do fórum seja feita à distância (Kralik et al., 2006). A confidencialidade dos dados no fórum online realizado por Moloney, Strickland, DeRossett, Melby e Dietrich (2006), por exemplo, foi assegurada pelo uso de firewalls e socket layers de segurança, além de os participantes terem recebido logins e senhas individuais para acessar o fórum.

De fato, os dispositivos de segurança mais citados nos artigos que relatavam a realização de grupos focais online assíncronos foram: (a) uso de senha (19 referências), (b) necessidade de o participante registrar-se no fórum para participar das discussões (6 referências), e (c) uso de login enviado pelos pesquisadores (5 referências). Esses dispositivos são fundamen- 
tais para evitar situações como a relatada por Adams, Rodham e Gavin (2005), na qual pessoas que não estavam participando do grupo focal interromperam a discussão postando mensagens agressivas. Os pesquisadores, que objetivavam investigar jovens que tivessem se automutilado, optaram por realizar o recrutamento em fóruns pré-existentes sobre esse tema e conduzir, ali mesmo, os grupos, dada a dificuldade de encontrar participantes com essa característica. Esses fóruns, contudo, eram abertos ao público, tornando impossível a proibição da entrada de não convidados no fórum.

\section{Participantes}

Este eixo de análise diz respeito: (a) ao meio de recrutamento dos participantes; (b) ao meio de devolução do TCLE aos pesquisadores; (c) à dificuldade de reunião dos participantes, se o grupo não fosse online; e (d) ao número de participantes.

Os participantes dos grupos focais online assíncronos analisados foram recrutados por conveniência. Apesar dos grupos terem sido realizados virtualmente, apenas 12 artigos mencionaram que o recrutamento fora exclusivamente via internet. Dez artigos referiram recrutamento realizado por outros meios que não a internet; oito referiram recrutamento por meios variados, como internet e telefone, por exemplo. No caso da pesquisa de Arbuckle et al. (2008) com pacientes que tinham pele oleosa, o recrutamento incluiu anúncios no jornal e na internet, bem como a procura de possíveis participantes em grupos de pacientes de dermatologistas. Os autores optaram pela variedade nos modos de recrutamento, dada a necessidade de garantir que a amostra contasse tanto com pessoas que estivessem consultando um médico por causa da sua pele oleosa quanto com pessoas com pele oleosa sem acompanhamento médico.

Quanto ao meio de devolução dos TCLE aos pesquisadores, a maioria dos 30 artigos (17) não apresentou comentários. Os outros mencionaram devolução por meios virtuais (6 artigos), consentimento implícito, ou seja, a postagem no grupo focal online fora considerada como consentimento (3 artigos), devolução por meios presenciais ( 2 artigos), pelo correio (1 artigo) ou de maneiras variadas ( 1 artigo). $\mathrm{O}$ consentimento por meios virtuais pode assumir diferentes formas. $\mathrm{O}$ participante pode, por exemplo, dar uma resposta positiva ao e-mail de convite (Lagan, Sinclair, \& Kernohan, 2011), ou pode dar um clique sobre o rótulo aprovo, apresentado após o texto do consentimento, no site da pesquisa (Johnson \& Lorig, 2011).
Já o consentimento implícito levanta questões acerca dos procedimentos éticos em ciência. No estudo de Kenny (2005), os participantes tinham a opção de se manter anônimos e liam informações sobre a pesquisa antes do início da mesma. No entanto, não havia consentimento, uma vez que eles eram avisados de que, ao postarem alguma mensagem no fórum, assumir-se-ia que tinham consentido em participar. Pode-se cogitar que a falta de um termo de consentimento faça com que o participante não avalie em profundidade as vantagens e desvantagens de sua colaboração. Em vista disso, sugere-se que, em estudos conduzidos na internet, essas desvantagens e o direito de abandonar a pesquisa sejam claramente apresentados e enfatizados aos participantes (Sharkey et al., 2011).

Ainda que se possam fazer algumas ressalvas ao uso dos grupos focais online assíncronos, quando empregam o consentimento implícito, são inegáveis as vantagens que tal técnica proporciona. Uma delas diz respeito à possibilidade de se ter uma amostra formada por participantes que dificilmente poderiam reunir-se de outro modo, seja pela distância, pelo custo ou por problemas de saúde que impeçam sua locomoção. De fato, dos 30 artigos analisados, 26 referiram que teria sido difícil reunir os participantes, caso o grupo não tivesse sido online e não tivesse usado comunicação assíncrona - o que permite que os membros do grupo participem no horário que lhes é mais conveniente. Utilizando o meio online e a comunicação assíncrona, Williams e Reid (2010) conseguiram realizar um grupo focal com 14 pessoas que tinham distúrbios alimentares severos e que frequentavam sites próanorexia. Como os membros do grupo não precisavam encontrar-se presencialmente e não tinham um horário pré-definido para participar, os pesquisadores conseguiram reunir pessoas dos cinco continentes. Apenas quatro artigos relataram grupos focais online realizados com pessoas que poderiam facilmente ter se encontrado presencialmente.

Em relação ao número de participantes dos grupos focais online assíncronos, observou-se que foi variado. Houve relatos de 4 até 38 participantes por grupo. A maior parte dos artigos (13) mencionou a realização de grupos focais com até 10 participantes; seis artigos citaram grupos de 11 a 16 membros, três citaram grupos de mais de 25 pessoas e oito não especificaram esse número. Kenny (2005), que descreveu um grupo online assíncrono com 38 membros, assinalou que a comunicação assíncrona permitiu que apenas um moderador facilitasse a discussão em um grupo desse tamanho, uma vez que as mensagens não eram envia- 
das todas no mesmo horário. Lagan et al. (2011) enfatizaram que o formato assíncrono possibilita que os grupos sejam grandes o suficiente para fornecer a diversidade de opiniões que o pesquisador busca.

Já Stjernsward e Ostman (2011), que realizaram um grupo focal com 16 pessoas, observaram vantangens e desvantagens de trabalhar com um grupo assíncrono considerado pequeno por eles. Segundo os autores, o pequeno número de participantes fez com que os usuários regulares fossem reconhecidos, criando um sentimento de familiaridade no grupo. Por outro lado, o grupo pode ter se tornado desinteressante, pois a participação de poucas pessoas fez com que a atividade do fórum não fosse muito intensa. De qualquer forma, ao planejar quantos componentes o grupo terá, é interessante que o pesquisador que trabalhará com grupos assíncronos considere um número maior do que o desejado. A ausência de um horário predeterminado para participar e a possibilidade de simplesmente deixar de enviar mensagens permite que os participantes fiquem mais à vontade para abandonar o estudo (Sharkey et al., 2011; Yu, Taverner, \& Madden, 2011).

\section{Moderadores}

Este eixo de análise aborda (a) o número de moderadores por grupo, (b) se estes eram os próprios pesquisadores ou não, e (c) as especificidades envolvidas na moderação de um grupo focal online assíncrono. Quanto ao número de moderadores, a maior parte dos artigos (21) referiu a presença de um moderador para cada grupo; cinco artigos mencionaram dois moderadores e quatro artigos não forneceram esse dado. Assim, é possível que a maioria dos autores concorde com a observação de que, no modelo assíncrono, um moderador é suficiente, inclusive para grupos numerosos (Kenny, 2005). No entanto, ressalta-se que $\mathrm{Yu}$ et al. (2011), embora tenham trabalhado com um grupo de 13 pessoas, acreditam que o uso de dois moderadores aumente a capacidade de monitoramento do grupo, permitindo encontrar sinais de desconforto nos participantes - aspecto importante quando a amostra inclui pessoas em situação de vulnerabilidade.

No que diz respeito à relação do moderador com a pesquisa, em 20 dos 30 artigos examinados foram os próprios pesquisadores que moderaram os grupos; em 4 artigos, o moderador fazia parte da equipe de pesquisa e, em 6 artigos, esse dado não foi citado. Nos quatro casos em que faziam parte da equipe de pesquisa, os moderadores compartilhavam alguma característica com os participantes dos grupos focais e esta- vam relacionados à cultura destes. McNaughton et al. (2008), por exemplo, para moderar um grupo de pais de pessoas que usavam dispositivos de comunicação alternativa e ampliada, escolheram alguém que também os utilizava e tinha experiência acadêmica com tais tecnologias de assistência. Parece que a opção por moderadores que têm algo em comum com os membros do grupo objetiva facilitar o estabelecimento de laços no grupo e aproveitar a experiência pessoal e acadêmica do moderador para a pesquisa.

Em relação às especificidades envolvidas na moderação de um grupo focal online assíncrono, destacaram-se: necessidade de experiência no uso da internet, de iniciativa para começar as discussões (Stjernsward \& Ostman, 2011) e de treinamento na condução de comunicação assíncrona (Lagan et al., 2011). Além disso, o moderador de um grupo assíncrono é responsável por contatar os participantes - sobretudo aqueles que ficam sem postar mensagens em algum tópico com o objetivo de estimulá-los a colaborar com a discussão (McNaughton et al., 2002; Stjernsward \& Ostman, 2011).

\section{Natureza dos tópicos discutidos}

Este eixo de análise enfoca o quanto os tópicos discutidos nos grupos focais online assíncronos eram delicados ou íntimos, visto que, segundo Kralik et al. (2006), os grupos virtuais seriam mais adequados para coletar impressões e opiniões relativamente simples e genéricas. Contrariando essa ideia, a maior parte dos artigos (19) referiu que os tópicos trabalhados eram delicados ou íntimos. Adams et al. (2005), por exemplo, consideraram que os grupos focais online assíncronos realizados com jovens que tinham se automutilado permitiram o acesso ao self dos participantes, que foram capazes de refletir sobre suas experiências. Os outros 11 artigos mencionaram discussões sobre tópicos que não eram delicados, tampouco íntimos.

\section{Equilíbrio entre as participações dos membros do grupo}

Este eixo de análise refere-se ao quanto as participações dos membros dos grupos focais online assíncronos foram equilibradas em termos numéricos e qualitativos. Dos 30 artigos examinados, 20 não continham essa informação. Dois artigos referiram que as participações dos membros do grupo foram equivalentes em termos numéricos. Finke et al. (2009), por exemplo, relataram que todos os participantes tinham postado comentários em todos os tópicos propostos. Por outro lado, foram contabilizados oito artigos que 
mencionaram não ter havido equilíbrio entre as participações, sobretudo quantitativamente. No caso do artigo de Hanley (2006), não houve referência às taxas de participação, mas à variação no comprimento das postagens: desde respostas de uma linha até respostas de vários parágrafos.

Há indícios, então, de que seja comum a flutuação nos índices de participação nas discussões em grupos focais online assíncronos. Uma das explicações para isso seria a variação na velocidade de adaptação dos diferentes participantes à interação online (Kralik et al., 2006). No grupo realizado por Williams e Reid (2010) - no qual alguns participantes contribuíram mais às discussões do que outros - alguns simplesmente responderam às perguntas do moderador, enquanto outros se comunicaram entre si e formaram suas próprias discussões. Este é um dado importante, pois, neste caso, torna-se papel do moderador evitar que alguns participantes dominem a discussão e que os outros se transformem em meros leitores do que está sendo produzido.

Para reduzir a flutuação nos níveis de participação, os pesquisadores que trabalham com grupos focais online assíncronos vêm utilizando algumas técnicas para ter maior controle sobre o grupo. $\mathrm{O}$ artifício mais citado (em 11 artigos) foi a postagem paulatina das questões, evitando que todos os tópicos de discussão ficassem online ao mesmo tempo e logo de início. Isso, segundo Adler e Zarchin (2002) e Walden e Bryan (2011), aumenta a probabilidade de todos os participantes responderem todas as questões, visto que os incita a elaborar uma resposta para cada tópico. Já no grupo relatado por Finke et al. (2009), os participantes foram requisitados a visitar o site de discussão pelo menos uma vez por semana, a responder às questões colocadas pelo moderador e a comentar ou responder às ideias e informações emitidas pelos outros participantes nos tópicos anteriores. Como se pode deduzir, esses artifícios acabam diminuindo a flexibilidade de participação na discussão, característica do modelo assíncrono.

\section{Anonimato}

Este eixo de análise diz respeito aos procedimentos, citados nos artigos, para que o anonimato dos participantes fosse mantido. A metade dos artigos analisados (15) não fez comentários sobre o anonimato dos participantes, 2 relataram grupos focais em que não houve anonimato e 13 relataram grupos em que houve. Destes 13, diversos referiram procedimentos para manter anônimos os participantes, como: (a) usar pseudônimos para divulgação dos dados, (b) requisitar aos membros do grupo que utilizassem endereços de $e$-mail diferentes dos seus usuais para entrar no fórum ou na lista de $e$-mails e (c) solicitar-lhes o emprego de um nick, isto é, um apelido ou pseudônimo. O simples uso desse último artifício, no entanto, não garante o anonimato dos participantes. Para isso, deve-se pedir para que eles escolham um nick que não exponha suas identidades e que não pode ser revelado a ninguém (Deggs, Grover, \& Kacirek, 2010; Walden \& Bryan, 2011). Outra recomendação é que se tenha cuidado com computadores que salvam senhas automaticamente, pois isso pode permitir que outros entrem no fórum ou na lista de e-mails (Stjernsward \& Ostman, 2011).

\section{Grupos Focais Online Síncronos}

Do total de artigos, $16(34,8 \%)$ relatavam o uso desse tipo de grupo focal.

\section{Ferramentas utilizadas}

No caso dos grupos focais online síncronos, os tipos de programa de computador mais utilizados para a sua execução foram os de conferência virtual e as salas de bate-papo, cada um citado em seis artigos, seguidos dos fóruns online, citados em dois artigos. Houve ainda dois artigos que não fizeram referência ao programa escolhido. As salas de bate-papo são oferecidas por sites. Alguns autores usaram salas disponibilizadas por sites relacionados à própria pesquisa ou pelo site de sua universidade. Alvarez, Cuenca, Noronha e Schor (2007), por exemplo, ao trabalharem com um grupo de pesquisadores usuários de uma biblioteca virtual, utilizaram uma sala de bate-papo de uma instituição de fomento à ciência. As salas de bate-papo podem estar sempre online e restringir ou não a entrada de usuários; nelas ocorre a troca de mensagens escritas entre os presentes na sala.

Já os programas de conferência virtual exigem que os participantes sejam convidados para uma conversa online, que fica automaticamente gravada em arquivo produzido pelo próprio programa. Os programas de conferência online citados nos artigos foram variados. Alguns autores mencionaram programas comumente utilizados por usuários da internet, como MSN e ICQ. Curiosamente, em 5 dos 6 estudos que citaram o uso de programas de conferência virtual, os participantes encontravam-se no mesmo local. Quando usados em outros campos da ciência, os grupos focais online síncronos não costumam ocorrer presencialmente, ou seja, cada componente encontra-se em um lugar dis- 
tinto. Contudo, nos referidos estudos, os membros do grupo estavam todos na mesma sala com os computadores ligados em rede. Os grupos relatados por Franklin e Lowry (2001), por exemplo, reuniram-se em uma sala da universidade equipada com 14 computadores em rede e uma tela de projeção. Nesta sala, os monitores dos computadores ficavam embutidos nas mesas, facilitando o contato visual entre os presentes, enquanto uma tela de projeção mostrava todas as mensagens enviadas. Conforme os autores, ainda que os participantes estejam na mesma sala, o anonimato permitido pelo ambiente virtual facilita a emissão de opiniões acerca de tópicos delicados.

No entanto, essa não foi a principal razão pela qual os pesquisadores optaram pela execução dos grupos no formato online; dos 5 estudos que empregaram programas de conferência virtual com participantes reunidos no mesmo local, apenas 1 abordou tópicos delicados ou íntimos. Alguns dos motivos sugeridos pelos autores relacionavam-se à facilidade para coletar e analisar dados e ao equilíbrio entre as participações de todos os componentes do grupo, propiciados pela sua realização online.

Chama atenção, também, que apenas 4 dos 16 artigos examinados mencionaram os dispositivos de segurança oferecidos pelos programas utilizados. Três deles citaram a necessidade de o participante registrar-se no programa para participar das discussões, sendo que dois destes também referiram que o grupo focal era protegido por senha. Em um dos casos, além disso, cada participante recebeu uma conta de $e$-mail individual para ser usada exclusivamente na pesquisa. $\mathrm{O}$ outro artigo mencionou que cada componente do grupo recebeu login enviado pelos pesquisadores. Quanto à segurança oferecida pelo programa usado para realização dos grupos focais online síncronos, Alvarez et al. (2007) referiram dificuldades para encontrar uma sala de bate-papo que possibilitasse o controle dos usuários, evitando a entrada de pessoas desconhecidas na conversa. Esses autores também citaram outra questão importante na escolha do programa a ser empregado: as facilidades que ele oferece para o pesquisador. Este deve certificar-se de que a sala de batepapo seja de fácil utilização e possua ampla capacidade de armazenamento, o que evita que parte da discussão seja apagada à medida que a página é preenchida (Abreu et al., 2009; Alvarez et al., 2007).

\section{Participantes}

Os participantes de todos os grupos síncronos referidos nos artigos foram recrutados por conveniência, exceto os dos grupos relatados por Blomberg et al. (2011). Estes pesquisadores utilizaram uma amostragem randomizada estratificada, para atingir heterogeneidade entre os grupos e, ao mesmo tempo, manter um grau de homogeneidade em cada grupo. Para recrutar os participantes, assim como foi observado em relação aos grupos focais online assíncronos, apenas sete artigos mencionaram que o recrutamento fora executado exclusivamente via internet. Os outros artigos referiram recrutamento realizado por outros meios que não a internet (5 artigos), por meios variados (2 artigos), ou não forneceram esse dado (2 artigos). Como muitos dos artigos relataram grupos online em que os participantes localizavam-se no mesmo recinto, já era esperado que boa parte deles mencionasse recrutamento não realizado pela internet. No entanto, mesmo os pesquisadores que trabalham com grupos online reunidos presencialmente parecem recorrer à internet para recrutar os participantes, dada a facilidade dessa ferramenta. Na pesquisa de Tyler et al. (2009), por exemplo, embora fossem estar na mesma sala, os componentes do grupo foram recrutados por meio de pôsteres afixados em universidades e $e$-mails. Esses pesquisadores aproveitaram, assim, as vantagens do contato virtual e do presencial.

Quanto ao meio de devolução dos TCLE aos pesquisadores, apenas 4 dos 16 artigos fizeram referência a essa questão. Dois artigos relataram devolução por meios virtuais, um por meios presenciais, e um de maneiras variadas. Chamou atenção a experiência relatada por Fox et al. (2007a) quanto à entrega e ao recebimento dos TCLE. Inicialmente, foi solicitado aos participantes que imprimissem o TCLE postado no site da pesquisa, pegassem a assinatura de um responsável e o devolvessem pelo correio. No entanto, frente ao pequeno retorno que obtiveram, os pesquisadores resolveram enviar o TCLE por $e$-mail. Tal processo, contudo, era muito demorado. Por fim, optaram por colocar o TCLE em um link na página de inscrição do site da pesquisa, para que, logo após o registro no site, o participante pudesse dar o seu consentimento. Observou-se, não obstante, que os mesmos autores, em outro artigo (Fox et al., 2007b), questionaram a devolução dos TCLE por meios virtuais, argumentando que jovens podem se fazer passar pelo seu responsável e dar o consentimento.

Ao contrário do que foi observado em relação aos grupos focais online assíncronos, o número de artigos que descreveram amostras que dificilmente poderiam se reunir se o grupo não tivesse sido online e síncrono (9 artigos) foi próximo ao número de artigos que des- 
creveram o oposto (7 artigos). Esse resultado era esperado, uma vez que 6 dos 16 artigos examinados relataram grupos focais online síncronos nos quais os participantes estavam no mesmo local. O grupo descrito por Cunningham, Deal, Neville, Rimas e Lohfeld (2006), por exemplo, ocorreu no laboratório da universidade à qual estavam vinculados os participantes, ou seja, o fato do grupo ter sido online e síncrono não foi o que permitiu sua reunião.

Quanto ao número de participantes dos grupos focais online síncronos, sete artigos referiram grupos de até 6 componentes; quatro artigos mencionaram que os grupos tinham de 6 a 12 participantes e dois artigos citaram mais de 12 membros. Houve ainda três artigos que não forneceram tal informação. Grupos de 6 a 12 pessoas são os mais comuns em pesquisas que usam a técnica dos grupos focais presenciais (Sirihal Duarte, 2007). No entanto, os grupos online síncronos relatados contaram, mais frequentemente, com menos componentes.

É provável que essa tenha sido uma opção dos pesquisadores. Contrariando a hipótese de que os grupos focais online poderiam ter mais participantes do que os presenciais (Schneider et al., 2002), alguns autores referiram que grupos focais online síncronos com muitos participantes podem ser difíceis de moderar e podem dificultar o diálogo entre os membros (Bordini \& Sperb, 2011; Fox et al., 2007a). Outra possível explicação para o trabalho com grupos de poucos componentes é a perda amostral envolvida nos grupos focais online síncronos. Poucos artigos forneceram esse dado, mas muitos mencionaram dificuldade, por parte dos pesquisadores, em agendar a discussão ou mesmo em realizá-la no horário combinado. Os empecilhos mais citados foram as diferenças de fuso horário e a falta de tempo e de comprometimento dos participantes (Alvarez et al., 2007; Bordini \& Sperb, 2011; Fox et al., 2007a). Para aumentar as chances de que os convidados conectem-se no horário combinado para a discussão, os autores aconselham: relembrar o horário a todos, dias antes e na véspera da sua realização; nesse lembrete, colocar o horário já adequado ao fuso do local em que o participante encontrar-se-á; evitar o agendamento de discussões em dias úteis; recrutar mais pessoas do que o necessário (Abreu et al., 2009; Fox et al., 2007a).

\section{Moderadores}

Quanto ao número de moderadores, a maior parte dos artigos (15) referiu a presença de um moderador para cada grupo focal online síncrono, assim como ocorreu nos grupos assíncronos. Apenas um artigo mencionou dois moderadores por grupo. Esse resultado, contudo, não deve levar à conclusão de que seja fácil moderar um grupo síncrono. A leitura integral dos artigos revelou que alguns dos grupos com apenas um moderador necessitaram de uma segunda pessoa da equipe de pesquisa para ajudar na sua execução. Dois artigos mencionaram que, além do moderador, é importante ter um observador para acompanhar a discussão e copiar as mensagens enviadas pelos participantes, caso o programa não o faça automaticamente (Abreu et al., 2009; Alvarez et al., 2007). Questões técnicas também fizeram com que alguns grupos síncronos contassem com a presença de um especialista no programa usado (Cunningham et al., 2006) ou com um facilitador para explicar a tecnologia aos participantes (Franklin \& Lowry, 2001).

Isso talvez se deva às especificidades envolvidas na moderação de grupos focais online síncronos. A rapidez com que se dá a comunicação síncrona exige que o moderador seja veloz na digitação e atento à simultaneidade das mensagens enviadas (Bordini \& Sperb, 2011; Fox et al., 2007a; Walsh, White, \& Greaney, 2009), o que pode fazer com que sejam necessárias mais pessoas para ajudar em outras tarefas das quais o moderador não poderia dar conta sozinho. Além disso, o moderador precisa dominar o programa utilizado e o estilo de discussão síncrona, bem como deve estar familiarizado com os artifícios empregados na comunicação virtual para compensar a falta de contato oral e corporal (Bordini \& Sperb, 2011; Fox et al., 2007a; Tyler et al., 2009). De fato, nos grupos focais online síncronos, a responsabilidade do moderador em manter o grupo focado no tópico de discussão parece maior, uma vez que os participantes tendem a se dispersar e que conversas paralelas são comuns (Abreu et al., 2009; Franklin \& Lowry, 2001).

No que diz respeito à relação do moderador com a pesquisa, em 7 dos 16 artigos examinados, esse dado não foi apresentado. Os outros nove artigos mencionaram que o moderador era um dos pesquisadores (6 artigos), um membro da equipe de pesquisa (1 artigo) ou não tinha relação com o estudo ( 2 artigos). Nestes últimos (Cunningham et al., 2006; Tyler et al., 2009), optou-se por um moderador profissional, especialista na condução de grupo focais e experiente no programa usado para a realização do grupo online síncrono.

\section{Natureza dos tópicos discutidos}

Dos 16 artigos analisados, 9 relataram grupos focais online síncronos em que os tópicos trabalhados 
não eram nem delicados e nem íntimos; os outros 7 artigos mencionaram discussões sobre tópicos que eram delicados ou íntimos. Em alguns casos, o fato do grupo focal ter sido online e síncrono contribuiu para que assuntos delicados e íntimos fossem facilmente revelados pelos participantes (Blomberg et al., 2011; Fox et al., 2007a; 2007b). Contudo, o maior número de artigos que relataram que os tópicos discutidos não eram delicados ou íntimos sugere que os pesquisadores têm optado pelo uso dos grupos focais online síncronos por outras vantagens que estes oferecem.

\section{Equilíbrio entre as participações dos membros do grupo}

Assim como observado em relação aos grupos assíncronos, dos 16 artigos que relataram grupos síncronos, a maior parte (13) não informava o quanto as participações dos membros tinham sido equilibradas. Dois artigos referiram que as participações foram equivalentes em termos numéricos e um artigo referiu o contrário. Contudo, mesmo Reid e Reid (2005), que mencionaram que o modelo online síncrono não garante o equilíbrio entre as participações, assinalaram que a comunicação virtual contribui para isso. Segundo os autores, isso pode ser atribuído ao anonimato, típico dos ambientes virtuais, que deixa todos mais à vontade para opinar.

\section{Anonimato}

No entanto, nem todos os grupos focais online síncronos relatados contaram com o anonimato dos participantes. Dos 16 artigos examinados, apenas 7 mencionaram que o anonimato caracterizou as discussões; 5 relataram grupos focais em que não houve anonimato e 4 não fizeram comentários sobre essa questão. Curiosamente, até mesmo alguns grupos não caracterizados pelo anonimato seguiram procedimentos para obtê-lo, provavelmente em busca das vantagens decorrentes da participação anônima. Graças a essa característica, os participantes ficam mais confiantes e menos inibidos, podendo assim relatar histórias íntimas e delicadas e ficando mais à vontade para serem sinceros em suas opiniões (Fox et al., 2007a; 2007b; Franklin \& Lowry, 2001; Blomberg et al., 2011).

$\mathrm{O}$ artifício mais comumente citado para manter anônimos os participantes foi o uso de nicks ou apenas do seu primeiro nome. Contudo, em grupos focais online em que os componentes encontram-se no mesmo local, isso não garante o anonimato. Ainda assim, Bordini e Sperb (2011), por exemplo, além de enviarem a cada participante um endereço de $e$-mail indivi- dual para que acessassem o programa de conferência online, solicitaram que todos escolhessem um nick, embora estivessem reunidos na mesma sala e estudassem na mesma escola. Franklin e Lowry (2001) foram os únicos a empregar um procedimento que promovia o anonimato em grupos focais online síncronos reunidos em um mesmo lugar. Em sua pesquisa, esses autores utilizaram uma sala especificamente projetada para a realização de discussões grupais, nas quais o teclado dos computadores fica embutido na mesa do computador, dificultando que se ouvisse e visse quem estava digitando.

\section{CONCLUSÃO}

O uso dos grupos focais online na pesquisa em psicologia é ainda incipiente. Nos últimos 10 anos, identificaram-se apenas 24 estudos que usaram tal técnica publicados em revistas de psicologia ou que tivessem um psicólogo como autor. Entretanto, essa técnica de coleta de dados apresenta diversas vantagens para o pesquisador. Os estudiosos do campo da psicologia e de áreas afins enfatizaram que os grupos focais online são econômicos, pois não exigem toda a infraestrutura dos presenciais, tampouco os custos e o tempo despendidos na transcrição dos dados (Fox et al., 2007a; Kenny, 2005; Tates et al., 2009; entre outros).

Outra particularidade dos grupos focais online, apontada como vantajosa em pesquisas, foi o anonimato. Embora a falta de interação face a face possa prejudicar o fluxo da discussão e a identificação de emoções (Abreu et al., 2009; Fox et al., 2007b; Yu et al., 2011; entre outros), também tem efeitos positivos. A ausência de pistas, como sinais corporais, típica da maior parte dos grupos analisados, diminui as chances de que os membros do grupo percebam diferenças de status entre si e a desaprovação dos seus comentários (Schneider et al., 2002). Isso, somado à impossibilidade de serem reconhecidos, graças ao uso de nicks, pode explicar a coragem e a desinibição dos participantes. Ressalta-se, todavia, que são necessários vários artifícios para garantir o anonimato nos grupos focais online.

$\mathrm{O}$ recrutamento e o consentimento online também foram considerados vantajosos pelos pesquisadores. Ainda assim, estes não têm recrutado e recebido os TCLE unicamente de maneira virtual, possivelmente por se sentirem inseguros. $\mathrm{O}$ recrutamento online prejudica a representatividade da amostra, já que seleciona apenas usuários da internet; ademais, faz com que não se saiba se os participantes são quem dizem ser (Fox et al., 2007b; Sharkey et al., 2011). De fato, es- 
tudo com jovens brasileiros encontrou que estes costumam modificar informações pessoais reveladas na internet (Dias \& La Taille, 2006). Da mesma forma, recomenda-se cuidado com o consentimento online. Segundo Sharkey et al. (2011), deve-se balancear o montante de informação fornecida aos convidados, pois o excesso de palavras pode levá-los a consentir sem ler o TCLE na íntegra.

Identificaram-se especificidades no uso dos grupos focais online assíncronos e síncronos. Na pesquisa em psicologia e em áreas afins, entre 2001 e 2011, os grupos assíncronos foram mais frequentes. Estes foram usados, geralmente, para abordar tópicos delicados ou íntimos e apresentaram vantagens como a possibilidade de incluir desde poucos até muitos componentes, usando apenas um moderador. Além disso, contaram com membros que dificilmente se reuniriam se a pesquisa não fosse online e assíncrona, relativizando a não representatividade atribuída às amostras dos grupos focais online. Já os grupos síncronos foram menos usados do que os assíncronos e, em geral, contaram com menos membros do que os grupos focais presenciais, inclusive. Ademais, abordaram menos tópicos delicados ou íntimos. A opção de alguns estudiosos pelo modelo síncrono pareceu advir da possibilidade de realizar uma discussão em tempo real, com duração similar a dos grupos focais presenciais, mas com as vantagens do anonimato e da economia de tempo e dinheiro.

Por fim, com base nos artigos examinados, fazem-se recomendações para o pesquisador em psicologia que pretende trabalhar com a técnica dos grupos focais online. O programa para a sua execução deve assegurar a confidencialidade dos dados produzidos e o moderador precisa dominar tal técnica. Também é importante que todos os participantes estejam igualmente familiarizados com o tipo de programa a ser usado. É provável que a maioria já seja usuária da internet via computador, mas alguns podem não conhecer o programa escolhido. Isso pode alterar a dinâmica das interações, sobretudo nos grupos síncronos, em que o participante mais hábil na digitação tende a dominar a discussão (Fox et al., 2007b; Franklin \& Lowry, 2001). Assim, o uso da técnica online parece mais seguro quando a internet é um contexto natural para os participantes (Alvarez et al., 2007).

Ressalta-se, contudo, que essas conclusões baseiam-se na análise de uma parcela da produção acadêmica, correspondente a artigos presentes em algumas bases de dados. Ainda assim, considera-se que os resultados obtidos servem de estímulo para uma maior abertura da pesquisa em psicologia à técnica dos grupos focais online, visto que mostraram que tal técnica permite a interação grupal e o acesso à intimidade dos participantes. Porém, por não se tratar de uma transposição dos grupos presenciais para o ambiente virtual, o pesquisador interessado em realizar um grupo focal online deve informar-se sobre suas particularidades.

\section{REFERÊNCIAS}

Abreu, N. R., Baldanza, R. F., \& Gondim, S. M. G. (2009). Os grupos focais on-line: Das reflexões conceituais à aplicação em ambiente virtual. Revista de Gestão da Tecnologia e Sistemas de Informação, 6, 23-42.

Adams, J., Rodham, K., \& Gavin, J. (2005). Investigating the "self" in deliberate self-harm. Qualitative Health Research, 15, 1293-1309.

Adler, C. L. \& Zarchin, Y. R. (2002). The "virtual focus group": Using the internet to reach pregnant women on home bed rest. Journal of Obstetric, Gynecologic and Neonatal Nursing, 31, 418-427.

Alvarez, M. C. A., Cuenca, A. M. B, Noronha, D. P., \& Schor, N. (2007). Saúde reprodutiva: Uma contribuição para avaliação de biblioteca virtual. Cadernos de Saúde Pública, 23, 2317-2326.

Arbuckle, R., Atkinson, M. J., Clark, M., Abetz, L., Lohs, J., Kuhagen, I., Harness, J., Draelos, Z., Thiboutot, D., BlumePeytavi, U., \& Copley-Merriman, K. (2008). Patient experiences with oily skin: The qualitative development of content for two new patient reported outcome questionnaires. Health and Quality of Life Outcomes, 6, 80-94.

Blomberg, K., Tishelman, C., Ternestedt, B. M., Tornberg, S., Leval, A., \& Widmark, C. (2011). How can young women be encouraged to attend cervical cancer screening? Suggestions from face-to-face and internet focus group discussions with 30year-old women in Stockholm, Sweden. Acta Oncologica, 50, $112-120$

Bordini, G. S. \& Sperb, T. M. (2011). O uso dos grupos focais online síncronos em pesquisa qualitativa. Psicologia em Estudo (Maringá), 16, 437-445.

Chase, L. \& Alvarez, J. (2000). Internet research: The role of the focus group. Library \& Information Science Research, 22, 357369.

Cunningham, C. E., Deal, K., Neville, A., Rimas, H., \& Lohfeld, L. (2006). Modeling the problem-based learning preferences of McMaster University undergraduate medical students using a discrete choice conjoint experiment. Advances in Health Sciences Education, 11, 245-266.

Deggs, D., Grover, K., \& Kacirek, K. (2010). Expectations of adult graduate students in an online degree program. College Student Journal, 44, 690-699.

Dias, A. C. G. \& La Taille, Y (2006). O uso das salas de bate-papo na internet: Um estudo exploratório acerca das motivações, hábitos e atitudes dos adolescentes. Interação em Psicologia, 10, 43-51. 
Finke, E. H., McNaughton, D. B., \& Drager. K. D. (2009). “All children can and should have the opportunity to learn": General education teachers' perspectives on including children with autism spectrum disorder who require AAC. Augmentative and Alternative Communication, 25, 110-122.

Fox, F. E., Morris, M., \& Rumsey, N. (2007a). Doing synchronous online focus groups with young people: Methodological reflections. Qualitative Health Research, 17, 539-547.

Fox, F. E., Rumsey, N., \& Morris, M. (2007b). "Ur skin is the thing that everyone sees and you cant change it!": Exploring the appearance-related concerns of young people with psoriasis. Developmental Neurorehabilitation, 10, 133-141.

Franklin, K. K. \& Lowry, C. (2001). Computer-mediated focus group sessions: naturalistic inquiry in a networked environment. Qualitative Research, 1, 169-184.

Hanley, T. (2006). Developing youth-friendly online counseling services in the United Kingdom: A small scale investigation into the views of practitioners. Counselling and Psychotherapy Research, 6, 182-185

Instituto Brasileiro de Geografia e Estatística - IBGE (2008). Acesso à internet e posse de telefone móvel celular para uso pessoal. Retirado de http://www.ibge.gov.br/home/estatistica/ populacao/acessoainternet2008/internet.pdf

Johnson, V. B., \& Lorig, K. (2011). The internet diabetes selfmanagement workshop for American indians and Alaska natives. Health Promotion Practice, 12, 261-270.

Kenny, A. J. (2005). Interaction in cyberspace: An online focus groups. Journal of Advanced Nursing, 49, 414-422.

Kralik, D., Price, K., Warren, J., \& Koch, T. (2006). Issues in data generation using email group conversations for nursing research. Journal of Advanced Nursing, 53, 213-220.

Lagan, B. M., Sinclair, M., \& Kernohan, W. G. (2011). What is the impact of the internet on decision-making in pregnancy? A global study. Birth Issues in Perinatal Care, 38, 336-345.

McNaughton, D., Light, J., \& Arnold, K. B. (2002). "Getting your wheel in the door": Successful full-time employment experiences of individuals with cerebral palsy who use augmentative and alternative communication. Augmentative and Alternative Communication, 18, 59-76.

McNaughton, D., Rackensperger, T., Benedek-Wood, E., Krezman, C., Williams, M. B., \& Light, J. (2008). "A child needs to be given a chance to succeed": Parents of individuals who use AAC describe the benefits and challenges of learning AAC technologies. Augmentative and Alternative Communication, 24, 43-55.

Moloney, M. F., Strickland, O. L., DeRossett, S. E., Melby, M. K., \& Dietrich, A. S. (2006). The experiences of midlife women with migraines. Journal of Nursing Scholarship, 38, 278-285.

Morgan, D. L. (1997). Focus groups as qualitative research. London: Sage.
Owen, S. (2001). The practical, methodological and ethical dilemmas of conducting focus groups with vulnerable clients. Journal of Advanced Nursing, 36, 652-658.

Reid, D. J. \& Reid, F. J. M. (2005). Online focus groups: An indepth comparison of computer-mediated and conventional focus group discussions. International Journal of Market Research, 47, 131-162.

Schneider, S. J., Kerwin, J., Frechtling, J., \& Vivari, B. A. (2002). Characteristics of the discussion in online and face-toface focus groups. Social Science Computer Review, 20, 31-42.

Sharkey, S., Jones, R., Smithson, J., Hewis, E., Emmens, T., Ford, T., \& Owens, C. (2011). Ethical practice in internet research involving vulnerable people: Lessons from a self-harm discussion forum study (Sharptalk). Journal of Medical Ethics, 37, 752-758.

Sirihal Duarte, A. B. (2007). Grupo focal online e offline como técnica de coleta de dados. Informação \& Sociedade: Estudos, $17,81-95$.

Stjernsward, S., \& Ostman, M. (2011). Illuminating user experience of a website for the relatives of persons with depression. International Journal of Social Psychiatry, 57, 375-386.

Tates, K., Zwaanswijk, M., Otten, R., Van Dulmen, S., Hoogerbrugge, P. M.; Kamps, W. A., \& Bensing, J. M. (2009). Online focus groups as a tool to collect data in hard-to-include populations: Examples from pediatric oncology. BMC Medical Research Methodology, 9, 1-8.

Tyler, I. V., Hau, M., Buxton, J. A., Elliott, L. J., Harvey, B. J., Hockin, J. C., \& Mowat, D. L. (2009). Canadian medical students' perceptions of public health education in the undergraduate medical curriculum. Academic Medicine, 84, 1307-1312.

Walden, P. R. \& Bryan, V. C. (2011). Speech-language pathologists' informal learning in healthcare settings: Behaviours and motivations. International Journal of Speech-Language Pathology, 13, 378-388.

Walsh, J. R., White, A. A., \& Greaney, M. L. (2009). Using focus groups to identify factors affecting healthy weight maintenance in college men. Nutrition Research, 29, 371-378.

Williams, S. \& Reid, M. (2010). Understanding the experience of ambivalence in anorexia nervosa: the maintainer's perspective. Psychology and Health, 25, 551-567.

Yu, J., Taverner, N., \& Madden, K. (2011). Young people's views on sharing health-related stories on the internet. Health and Social Care in the Community, 19, 326-334.

\section{Nota:}

1 Este artigo foi financiado pela Coordenação de Aperfeiçoamento de Pessoal de Nível Superior (CAPES) e pelo Conselho Nacional de Desenvolvimento Científico e Tecnológico (CNPq). 